\title{
EL PROYECTO ACADÉMICO DE TRABAJO APLICADO (PAT). DEL DERECHO A LOS DERECHOS
}

\begin{abstract}
Autores: Juan Carlos Berrocal Durán ${ }^{1}$
Correspondencia: en: juan.berrocal@curnvirtual.edu.co

http://scienti1.colciencias.gov.co:8081/cvlac/visualizador/generarCurriculoCv.do?cod_rh=00003516 44

\section{RESUMEN}

Luego de su instauración el Proyecto Académico de Trabajo Aplicado (PAT) en el Programa de Derecho en la Sede Barranquilla fue sometido a una evaluación, con respecto a sus avances y desarrollos. La práctica evaluativa permitió establecer que había que hacer significativos cambios al proceso, adecuándolo a la dinámica actual del Derecho. Surge EL PROYECTO ACADÉMICO DE TRABAJO APLICADO (PAT). DEL DERECHO A LOS DERECHOS, como una alternativa de evidencia para el trabajo que realizan los estudiantes, desde el Primero hasta el último de los semestres.

El objetivo es evaluar el proyecto académico de trabajo aplicado (pat). El método empleado es Cualitativo, y el tipo de investigación es explicativa. En conclusión el proceso fue excelente por los resultados obtenidos, pero se replantea la forma de asumir los Pat colectivos
\end{abstract}

\section{Palabras Claves} Jurídico

Proyecto Académico de Trabajo (PAT), Contexto, Reflexión, La Escuela del Realismo

\begin{abstract}
After its establishment Academic Applied Project Work ( PAT ) Program at Headquarters Barranquilla law underwent an evaluation regarding their progress and developments. The evaluation practice established that had to make significant changes to the process, adapting to the current dynamics of law. Surge ACADEMIC WORK PROJECT APPLIED (PAT ). THE RIGHTS LAW , as an alternative of evidence to the work done by students from the first to the last semester . The objective is to evaluate the academic project applied work (pat). The method is qualitative, and the type of research is explanatory. In conclusion the process was excellent for results, but how to take collective Pat rethinks
\end{abstract}

\section{Keywords}

\footnotetext{
1 Abogado. Especialista en Derecho Laboral. Administrador Agropecuario. Candidato a Magíster en Derecho de la Universidad del Norte. Docente Programa de Derecho Barranquilla de la Corporación Universitaria Rafael Núñez.
} 
Vol. 6, No. 1 Diciembre de 2014 pp. 17 - 30 Jurídico

Proyecto Académico de Trabajo (PAT), Contexto, Reflexión, La Escuela del Realism

\section{INTRODUCCIÓN}

La preocupante pregunta que nos hacen los estudiantes de Derecho en la Corporación Universitaria Rafael Núñez Sede Barranquilla, debe ser similar a la que en su turno debieron asumir prestigiosos docentes en otras universidades del país: ¿cuándo es que voy a aprender lo que es ser un abogado de verdad? Como ya las universidades no están para suministrar respuestas estilo "aguas tibias", en esta seccional se ha arrancado con un proyecto que implica una razón de aprendizaje en la misma práctica, un estilo y dinámica de docencia diferente, y la puesta en escena de la combinación de teoría y práctica en el contexto.

A tal propuesta se le ha denominado EL PROYECTO ACADÉMICO DE TRABAJO APLICADO (PAT). DEL DERECHO A LOS DERECHOS, con los siguientes objetivos:

- Integrar al estudiante con la problemática jurídica, de la vivencia cotidiana, en los diferentes escenarios sociales.

- Permitir la aplicación inmediata del conocimiento teórico, adelantando actividades de solución real. Propicia el diseño de documentos monográficos (investigación descriptiva socio jurídica) a partir de casos concretos.

- Favorecer la articulación del PAT con las líneas de investigación de la CURN.

- Promocionar la UNIVERSIDAD en diferentes escenarios sociales.

- Desarrollar habilidades profesionales en el Estudiante de Derecho.

Todo ello en una dinámica que se oriente por un constante diálogo entre diversos interlocutores es "posible un proceso dialéctico de reflexión - acciónreflexión- desde una perspectiva democratización de conocimiento partiendo de la idea de que alguien extiende algo a otro/a" (Cedrón, C, Gámez, A, Montenegro, $M$ 
Vol. 6, No. 1 Diciembre de 2014 pp. 17 - 30

\& Tempori $L$ ). Esta situación plantea que se recurra al siguiente interrogante: ¿es

el Derecho una Ciencia secular?

\section{MATERIALES Y MÉTODOS}

Metodología de la Investigación: Cualitativa, adscrita a la línea de investigación línea sociedad, política y tipo de investigación fue: Explicativa.

Población estudiada: Estudiante de la Corporación Universitaria Rafael Nuñez en la sede Barranquilla, en el segundo periodo 2014

\section{RESULTADOS Y DISCUSIÓN}

En el campo del Derecho, como en otras disciplinas, es frecuente la secularización del saber, el conocimiento sobre el Derecho y los derechos son celosamente custodiados por las instituciones jurídicas, quedando la población alejada y ajena a las leyes y reglas de juego de este campo aun cuando rigen para todos y todas (Cedrón, 2010), cuando a todos les compete.

Con esta propuesta que se vislumbra como El componente de "La extensión del aula en la práctica jurídica de casos concretos" del PAT APLICADO, los estudiantes estarán en capacidad de desarrollar los siguientes las siguientes competencias:

- Integración de un profundo conocimiento teórico del Derecho con la práctica profesional y las habilidades propias del jurista.

- Alto grado de capacidad analítica y argumentativa, a partir de la discusión de casos hipotéticos y sentencias judiciales.

- Marcada aptitud para la resolución creativa de los problemas jurídicos, fomentada por la redacción de decenas de informes, dictámenes y escritos. 


\section{Vol. 6, No. 1 Diciembre de 2014 pp. 17 - 30}

- Disposición para adaptarse rápidamente a los constantes cambios del mundo del Derecho.

- Capacidad de promoción en forma permanente de debates interesantes, enriquecedores y de alto nivel jurídico.

- Amplitud para la comprensión de temas abordados en el ejercicio profesional.

- Mayor seguridad ante el cliente, que le permite un mejor desenvolvimiento en la solución de conflictos.

- Mayor predisposición para aceptar casos más complejos, con una visión más amplia que le permite tomar decisiones más acertadas.

El Proyecto Académico de Trabajo se estructura metodológicamente en dos componentes estratégicos para la construcción del conocimiento, siguiendo las orientaciones de las Escuelas que con mayor presencia han contribuido con la enseñanza del derecho.

- La Escuela Jurídica para la promoción de los derechos. Esta Escuela se destaca por su interés en acercar al estudiante con el discurso jurídico haciendo la extensión del DERECHO a las aulas básica de primaria y secundaria. Una manera de dinamizar la intervención de los estudiantes consiste en agruparlos en colectivos donde intervengan estudiantes de primer, segundo y decimo semestre.

En este sentido, el estudiante se convierte en agente de democratización del conocimiento, entenderá el proceso de compartir un saber a otro, coconstruyendo en esta interacción, conocimiento jurídico. 
Vol. 6, No. 1 Diciembre de 2014 pp. 17 - 30

Mediante convenios de alianza estratégica pedagógica, los estudiantes de la CURN, adelantarán cursos de formación en DERECHOS en instituciones educativas de básica primaria y secundaria, en los siguientes temas:

- Cátedra de Derechos Humanos

- Cátedra de la Igualdad de genero

- Cátedra para la paz

- Cátedra de democracia y participación ciudadana

En este sentido la transformación de la realidad social están sujetas a la voluntad del individuo por cuanto se cree que las condiciones están dadas en forma evidente para el cambio social, y que la educación será su órgano maestro. Son saludables y para un profundo pensar las palabras que expresadas por Gabriel García Márquez pretenden situar a la educación en el lugar que le corresponde:

Una educación desde la cuna hasta la tumba, debe ser inconforme y reflexiva, que nos inspire un nuevo modo de pensar y nos incite a descubrir quiénes somos en una sociedad que se quiera más que así misma. Que aproveche al máximo la creatividad inagotable y conciba una ética y tal vez una estética para nuestro afán desaforado y legítimo de superación personal. (García Márquez, G, 1996, Banco de la República)

Con el fin de obtener las memorias que sirvan de referente de investigación académica al finalizar el semestre y una vez cumplidas las horas de capacitación "cátedras", los estudiantes de cada equipo de trabajo presentarán (experiencias, logros, recomendaciones y conclusiones) en un informe descriptivo con todos los acápites de un trabajo académico de investigación.

El informe descriptivo será sustentado por cada grupo y se entregará impreso y en medio magnético con todos los requisitos del PAT. 
La evaluación académica debe ser sistemática y debe tomar en cuenta el desarrollo armónico de facultad del estudiante, la información necesaria para el ejercicio eficaz, las habilidades de operación, y en "general los contenidos que integran los grandes ejes del plan de estudios" (Damián J. \& Montes E. 2011).

Con el fin de obtener las memorias que sirvan de referente de investigación académica al finalizar el semestre y una vez cumplidas las horas de capacitación "cátedras", los estudiantes de cada equipo de trabajo presentarán (experiencias, logros, recomendaciones y conclusiones) en un informe descriptivo con todos los acápites de un trabajo académico de investigación.

El informe descriptivo será sustentado por cada grupo y se entregará impreso y en medio magnético con todos los requisitos del PAT.

- La extensión del aula en la práctica jurídica de casos concretos. El objetivo de esta Escuela consiste en acercar al estudiante a la realidad profesional, a través de la aplicación de los conocimientos teóricos, en una actividad práctica, como parte integral de su formación académica. Intervienen estudiantes de cuarto, quinto, sexto, séptimo, octavo, noveno y décimo semestre, agrupados en cinco estudiantes en equipos inter-semestre ( $3^{\circ}$ y $\left.7^{\circ}\right)$, ( $4^{\circ}$ y $\left.8^{\circ}\right)$, ( $5^{\circ}$ y $\left.9^{\circ}\right)$, (6ํㅜ y $\left.10^{\circ}\right)$. El Extensionismo Jurídico, como una actividad de reflexión-acciónreflexión cíclica y permanente desde y para la práctica social, efectuada por diversos actores desde sus perspectivas y metodologías pertinentes, con el objeto de facilitar transformaciones sociales, particularmente en el ámbito del conocimiento, ejercicio y exigibilidad de todos los derechos. A propósito del Extensionismo Jurídico es la ciencia del DERECHO y de los DERECHOS que está circunscrita a los abogados, se convierte en imperiosa necesidad que estos asuman la investigación SOCIO JURÍDICA en su conjunto, y se conviertan estos estudios en canales de promoción de derechos ciudadanos. 
Los estudiantes abordaran problemáticas reales, y casos concretos, para dar tratamiento y buscar la solución jurídica, así:

- Derechos de petición con representación, -Seguridad Social en Pensión

-Seguridad social en Salud

-Servicios públicos domiciliarios

-Servicios financieros y bancarios

- Acciones de tutela

- Acciones populares y de grupo

- Conciliaciones de pequeñas causas

La finalidad primordial de la atención de casos concretos por parte de los estudiantes, organizados en equipos de trabajo e investigación, es hacer actividades complementarias en la formación de los Abogados, proporcionándoles escenarios de perfeccionamiento, y adiestramiento práctico para el futuro ejercicio del DERECHO, así como herramientas para maximizar la capacidad de innovación jurídica.

Con este componente "La extensión del aula en la práctica jurídica de casos concretos" del PAT el estudiante estará en capacidad de desarrollar las siguientes habilidades:

- Disposición para adaptarse rápidamente a los constantes cambios del mundo del Derecho.

- Capacidad de promoción en forma permanente de debates interesantes, enriquecedores y de alto nivel jurídico.

- Amplitud para la comprensión de temas abordados en el ejercicio profesional.

- Mayor seguridad ante el cliente, que le permite un mejor desenvolvimiento en la solución de conflictos. 
- Mayor predisposición para aceptar casos más complejos, con una visión más amplia que le permite tomar decisiones más acertadas.

La evaluación del PAT APLICADO, en el componente La Extensión del Aula en la Práctica Jurídica de casos concretos, se realizará de la siguiente forma:

- Primer corte el Docente encargado del PAT, evaluará un anteproyecto por escrito presentado los estudiantes del equipo de trabajo.

- Segundo corte el Docente encargado del PAT, evaluará el desarrollo de los objetivos, y los documentos de la acción impetrada. Presentada por los estudiantes de cada equipo de trabajo, el cual debe ser sustentado por los estudiantes.

- Tercer corte el Docente encargado del PAT, evaluará el PAT a través de un informe descriptivo final y la sustentación. En el informe final se tendrá como anexo el documento de la acción impetrada y la resolución del caso.

En esta misma sintonía entra en juego los postulados de la Ley 583 de 2000 con la modificación de los Artículos 30 y 39 del Decreto 196 de 1971, que establecen que los estudiantes mientras pertenezcan al Consultorio Jurídico de las Facultades de Derecho oficialmente reconocidas podrán litigar en causa ajena en los siguientes asuntos, actuando como abogados de pobres, interviniendo:

1. En los procesos penales de que conocen los Jueces Penales municipales y los Fiscales delegados ante estos, así como las autoridades de Policía, en condición de apoderado de los implicados.

2. En los Procesos Penales de competencia de la Jurisdicción ordinaria, como representantes de la parte Civil.

3. De oficio en los Procesos Penales como voceros o defensores en audiencia. 
Vol. 6, No. 1 Diciembre de 2014 pp. 17 - 30

4. En los Procesos Laborales, en que la cuantía de la pretensión no exceda los 20 salarios mínimos legales mensuales vigentes y en las diligencias administrativas de conciliación en materia Laboral.

5. En los Procesos Civiles de que conocen los Jueces Municipales en única instancia.

6. En los procesos de alimentos que se adelantan ante los Jueces de Familia

7. De oficio en los procesos ${ }^{*}$ disciplinarios de competencia de la Personería Municipales y la Procuraduría General de la Nación.

8. De oficio en los Procesos ${ }^{\star \star}$ de responsabilidad Fiscal de Competencia de las Contralorías Municipales, Distritales, Departamentales y General de la República.

9. De oficio, en los procesos ${ }^{\star * *}$ administrativos de carácter sancionatorio que adelanten las autoridades administrativas, los organismos de control y las entidades constitucionales autónomas (Colombia. Corte Constitucional, 2011)

La propuesta se fundamenta en una Escuela (de las tres que tienen vigencia actualmente: La escuela del realismo estadounidense, con los siguientes adelantos:

Como precursor de los realistas estadounidenses se puede mencionar a Oliver Wendell Holmes Jr., quien consideraba que el derecho no es otra cosa que las profecías de cómo los jueces resolverán los asuntos jurídicos. Su principal representante es Karl Lleweltyn. Otros autores destacados son Roscoe Pound, Jerome Frank, Thurman Arnold, Félix S. Cohen, y B. N. Cardozo. Todos ellos son de la rama del Derecho y no filósofos. Como puntos comunes de este movimiento,

\footnotetext{
* Corte Constitucional numeral declarada condicionalmente exequible por la Corte Constitucional mediante Sentencia C-143 de fecha 7 de febrero de 2011, MP DR. JOSE GREGORIO HERNANDEZ GALINDO, siempre que los estudiantes que actúen en desarrollo ejerzan el Derecho bajo la supervisión, la guía y el control de las instituciones educativas a las cuales pertenecen.

${ }^{* * *}$ Ibid

****Ibid
} 
los abanderados siempre rechazaron el término "escuela", pues lo concebían como una concepción instrumental del derecho, como un medio para fines sociales. Otros puntos en común eran una visión dinámica de la sociedad y las instituciones; desconfianza en las reglas del método jurídico tradicional (Wikipedia 2014); rechazo de la teoría imperativista de las normas; valoración de las normas por sus efectos; y un enfoque del derecho desde la perspectiva de los casos y de los problemas reales que se presentan.

Fue en la primera mitad del siglo XX cuando se sintió el surgimiento y apogeo del Realismo Jurídico Norteamericano. Se trata de aglutinar con este nombre a las teorías de un grupo de juristas estadounidenses, que no formaron escuela según la academia tradicional, pero con un marcado reconocimiento en los libros de Historia del Derecho como parte del mismo movimiento.

Como máximo representante y como precursor de los realistas norteamericanos ha sido identificado a Oliver Wendell Holmes Jr., cuya obra fue considerada con una notoria influencia en los posteriores trabajos de dichos teóricos. A Holmes se le identifica como realista, a pesar de haber desarrollado su doctrina en una época anterior a la que se reconoce como propiamente realista.

El Realismo Jurídico Norteamericano dicen las investigadoras Evelyn Patricia Campos Flores y Bárbara Sepúlveda Hales

Logra tener éxito en los Estados Unidos, porque tiene el propósito de alcanzar una reforma legal liberalizadora, que en algunos casos sería reforma de índole constitucional y en otros de tipo legal. Sin embargo, una de sus principales consecuencias resulta ser el provocar un profundo cambio en la manera de pensar el Derecho para todos los involucrados. Jueces, abogados, juristas, académicos, resultan inspirados por una fuerte crítica, la compartieran o no, y se ven impulsados a modificar y revisar sus propias teorías y prácticas. (2013) 
Aunque declinó su fuerza luego de la Segunda Guerra Mundial, la influencia del Realismo Jurídico ha alcanzado a la corriente llamada "estudios críticos del derecho" (con Duncan Kennedy y Roberto Unger), a la teoría jurídica feminista y al análisis económico del derecho (entre los cuales se destacan autores como Richard Posner y Richard Epstein.

Sobre el desarrollo del Pat colectivo este tuvo ventajas y desventajas. Entre las primeras se encuentra las siguientes

Tabla 1. Ventajas y Desventajas

\begin{tabular}{|l|l|}
\hline \multicolumn{1}{|c|}{ Ventajas } & \multicolumn{1}{|c|}{ Desventajas } \\
\hline $\begin{array}{l}\text { Formación de un ser integral. Con } \\
\text { muchas ganas de mejorar y hacer las } \\
\text { cosas bien }\end{array}$ & $\begin{array}{l}\text { Algunos grupos no se han sintonizado, } \\
\text { con el cambio de paradigma }\end{array}$ \\
\hline $\begin{array}{l}\text { Hoy en día ellos están realizando sus } \\
\text { trabajos (no los mandan hacer) }\end{array}$ & $\begin{array}{l}\text { Se observa resistencia en algunos } \\
\text { grupos. }\end{array}$ \\
\hline $\begin{array}{l}\text { Luchan con sus temores al enfrentarse } \\
\text { a un usuario }\end{array}$ & $\begin{array}{l}\text { El estudiante no tiene confianza en sí } \\
\text { mismo }\end{array}$ \\
\hline $\begin{array}{l}\text { - Personas muy renacidas al cambio } \\
\text { entraron por la nueva metodología }\end{array}$ & Deficiencia en los poderes \\
\hline - Se fortalece la colectividad & Mala presentación de la demanda \\
\hline $\begin{array}{l}\text { Se perfila al estudiante como } \\
\text { procesalista del Derecho al realizar } \\
\text { estudios de caso }\end{array}$ & \\
\hline
\end{tabular}

Nombre de la fuente. Sistematización de Docentes y Estudiantes

Cada uno de los aspectos que sirvieron para la organización de los grupos de interesados se expone a continuación:

\section{Organización de grupos}

- No ha sido complicada la organización de los grupos. Aun cuando el encuentro con grupos diferentes les ha causado una conmoción. 
- Se observa el miedo del estudiante en el - saber y saber hacer. Sobre todo con un verdadero caso

\section{Trabajo de los grupos. Encuentro entre grupos}

- Algunos si cumplen con esta función (ser, saber y saber hacer)

- Otros le falta compromiso en el ser, saber y saber hacer

- La evaluación docente, la tutoría se debe evidenciar en la calidad del trabajo

\section{Consultorio jurídico}

- Ha encontrado un espacio que se ha reavivado palabras del director del consultorio jurídico (Dr. Fernando Barrero)

- Seguir insistiendo en el proceso todos los días aquí es donde vamos a evidenciar el ser integral.

- Presenta unos poderes mal hechos, no conocen de competencia de los estudiantes de consultorio jurídico.

\section{Biblioteca}

- Se han disparado las consultas de textos, base de datos y se aprovechan mejor las instalaciones. Según julio de la hoz (Director de biblioteca) los estudiantes antes se retiraban de la biblioteca a las 9 am hoy lo hacen a la $1 \mathrm{pm}$

- No todos los grupos lo hacen

- Percibo un cambio de actitud, con la limitante de que no saben cómo afrontar la utilización del texto, se inhiben ante la ficha bibliográfica

\section{Tutorías}

- La Tutorías se fortalece

- La utilización de los formatos de tutoría por parte del docente

\section{CONCLUSIONES}


En conclusión el proceso fue excelente por los resultados obtenidos, en cuanto se fortalecen las competencias del abogado en el ser, hacer, saber hacer. Pero se replantea la forma de asumir los Pat colectivos es decir los de primero con primero, los de segundo con segundo, los de tercero con los de tercero y así sucesivamente cada grupo seguirá formando grupos de 3 o de 5 estudiantes. De primero a sexto semestre se realizaran investigaciones desde la filosofía del derecho, la teoría del derecho y la sociología jurídica en cuanto a la justicia, validez y eficacia de la norma jurídica y a partir de séptimo a decimo semestre con un tipo de investigación de estudio de caso con la construcción de líneas jurisprudenciales, además le corresponde a estos estudiantes presentar una verdadera demanda.

\section{BIBLIOGRAFÍA}

Bustos. L. (14 de agosto de 2012). Realismo conductista. 14 de agosto de 2012, de Filosopi Sitio web: http://filosophiy.blogspot.com/2012/08/realismoconductista.html

Campos. E. \& Hales. B. (Septiembre 2013). El Realismo Jurídico Norteamericano: Escuela de Derecho. En Tesis de Grado (108). Universidad de chile facultad de derecho Sitio web: http://tesis.uchile.cl/bitstream/handle/2250/115314/decampos_e.pdf?sequence $=1$

Congreso de la república. (2000). LEY 583 DE 2000. Noviembre 15 de 2014, de Diario Oficial No. 44.042, del 13 de junio de 2000 Sitio web:

http://www.mineducacion.gov.co/1621/articles-105018_archivo_pdf.pd

Corte constitucional (Colombia) MP Dr. José Gregorio Hernández Galindo. (2011).Sentencia C-143 de fecha 7 de febrero de 2011. Noviembre 15 de 2014, Sitio web:

http://corteconstitucional.vlex.com.co/vid/43614382?_ga=1.112422727.21233 $0 \quad 6126.1407164357$

Cedrón, C, Gámez, A, Montenegro, M \& Tempori L. (SF). Estudio de Caso. El Agora, 1, 48. Noviembre 25 de 2014, Sitio web: file://C:/Documents\%20and\%20Settings/CONSULTA/Escritorio/Estudio-deCaso-Extensionismo.pdf 
Damián J. \&

Vol. 6, No. 1 Diciembre de 2014 pp. 17 - 30 Superior Universitario en Administración. Un Auto diagnóstico a través del Modelo CIPP. 1, 4, 10. Recuperado en Noviembre 15 de 2014, Sitio web: http://www.rinace.net/riee/numeros/vol4-num1/art9_htm.html

García. M. (SF). POR UN PAIS AL ALCANCE DE LOS NIÑOS. Noviembre 15 de 2014, de Banco de la República Sitio web:

http://www.banrepcultural.org/blaavirtual/educacion/etnoeduc/etno9.htm

Presidente de la República de Colombia. (1971). Decreto 196 de 1971 Por el cual se dicta el estatuto del ejercicio de la abogacía. Noviembre 15 de 2014, Universidad Sergio Arboleda Sitio web:

http://www.usergioarboleda.edu.co/derecho/estatuto_ejercicio_abogacia.pdf

Wikipedia. 18 julio 2014. Realismo jurídico. En Wikipedia (1, 2) http://es.wikipedia.org/wiki/Realismo_jur\%C3\%ADdico: Wikipedia. 